\title{
Revealing the nature of non-equilibrium phase transitions with quantum trajectories
}

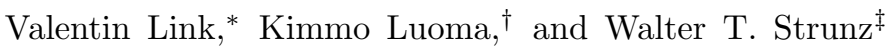 \\ Institut für Theoretische Physik, Technische Universität Dresden, D-01062,Dresden, Germany
}

(Dated: June 9, 2022)

\begin{abstract}
A damped and driven collective spin system is analyzed by using quantum state diffusion. This approach allows for a mostly analytical treatment of the investigated non-equilibrium quantum many body dynamics, which features a phase transition in the thermodynamical limit. The exact results obtained in this work, which are free of any finite size defects, provide a complete understanding of the model. Moreover, the trajectory framework gives an intuitive picture of the two phases occurring, revealing a spontaneously broken symmetry and allowing for a qualitative and quantitative characterization of the phases. We determine exact critical exponents, investigate finite size scaling, and explain a remarkable non-algebraic behaviour at the transition in terms of torus hopping.
\end{abstract}

Introduction Many important models from the early years of quantum optics, like the Dicke model [1, have experienced renewed interest. The reason being the availability of new experimental platforms. First and foremost, the field of ultra cold atomic gases allows an unprecedented level of control and tunability, bringing relatively simple but physically rich quantum optical models within the reach of current state of the art experiments [2 5]. The Dicke model, for example, can be realized for a wide range of parameters covering different phases of the system [6, 7]. Similar models are studied in the context of quantum magnetism [8] 10. Experiments are often performed under interesting non-equilibrium conditions where the interplay of driving and dissipation determines a stationary state of the system in absence of detailed balance 11 13. The dissipation stems from interactions with an environment which in many cases cannot be avoided. If the driven open system exhibits a phase transition upon tuning the system parameters, the non-equilibrium stationary state, rather than the ground state of the Hamiltonian, undergoes a nonanalytical change. This poses a formidable challenge for the theoretical treatment of such a system. The understanding of phase transitions in driven dissipative quantum many body systems is still developing [14-16], as such problems, in general, can be tackled only approximately [17.

Analytically soluble models containing the relevant physics are highly desirable and of great value for exploring in great detail new phenomena arising in this field. A recent trend has been to describe driven dissipative systems in the language of non-equilibrium quantum field theory [3, 11. There, a path integral formalism for Gorini-Kossakowski-Sudarshan-Lindblad (GKSL) master equations [18, conceptually similar to the Keldysh functional integrals, are used [11. Such path integral representations of dissipative propagators were earlier studied in a quantum optics setting in [19].

Alternatively, quantum trajectory methods from the field of quantum optics 20, 21, such as quantum state diffusion [20, also provide an efficient and transparent the- oretical framework. They can contribute to a detailed understanding of non-equilibrium quantum physics, as we will demonstrate in this Letter. In particular, the localization property of quantum state diffusion in the long time limit 22 is useful for the qualitative and quantitative analysis of non-equilibrium phase transitions, since this allows a direct observation of the different character of distinct phases. Quantum trajectories have been used successfully in 4 to analyze a generalization of the Dicke model. In the present Letter we consider a model similar to the so-called cooperative resonance fluorescence model introduced in the 1970s [23 25]. The latter recently received attention because it features a particularly interesting phase transition which is difficult to characterize [14, 26]. We here show that our model can be treated exactly within a quantum trajectory approach. This gives a clear picture of the different phases, helps to identify a broken symmetry, allows for an analytical, rather than numerical, determination of critical exponents and explains the peculiar critical behaviour.

Model We consider the following master equation of GKSL form [18 for a driven damped spin- $j$ system

$$
\begin{aligned}
\partial_{t} \rho= & -\mathrm{i} \omega\left[J_{x}, \rho\right]+\frac{\kappa}{j}\left(J_{+} \rho J_{-}-\frac{1}{2}\left\{J_{-} J_{+}, \rho\right\}\right) \\
& +\frac{\kappa}{j}\left(J_{z} \rho J_{z}-\frac{1}{2}\left\{J_{z}^{2}, \rho\right\}\right),
\end{aligned}
$$

where $J_{z}, J_{ \pm}=J_{x} \pm \mathrm{i} J_{y}$ are the spin operators. Apart from the additional $J_{z}$ dissipator this coincides with the cooperative resonance fluorescence model $23,25,27,28$. It can describe $j$ spin- $\frac{1}{2}$ systems undergoing collective driving and collective damping. Following the proposals in [14, 26] this model could be realized experimentally with cold atoms. Interestingly, in theoretical models for engineered atomic spin devices used to describe tunneling spectroscopy of atomic magnets on metallic surfaces, a similar GKSL dissipator appears naturally [8-10]. A phase diagram of this model was examined in [10].

The stationary state of (1) features a phase transition in the thermodynamic limit $j \rightarrow \infty$ as the parameter $\lambda=\frac{\omega}{\kappa}$, measuring the relative strength of coherent drive 


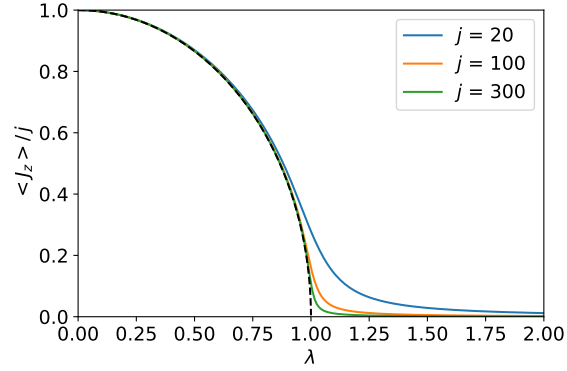

FIG. 1: $J_{z}$ expectation value in the steady state of master equation (1) as a function of the parameter $\lambda$ for different values of $j$. The dashed line is the asymptotic curve for $j \rightarrow \infty$ from $(9)$.

to dissipation, is changed. For strong damping $\lambda<1$ the steady state has a finite $J_{z}$ expectation value. If instead $\lambda>1$ the $J_{z}$ expectation value is zero, see Fig. 1 .

It is important to stress that Eq. (1) has a discrete symmetry. It is invariant under mirror reflection given by $J_{x} \rightarrow-J_{x}$ followed by complex conjugation. The crucial role of this symmetry for the phase transition becomes apparent when we add a symmetry breaking term of the form $\omega_{z} J_{z}$ to the Hamiltonian. As shown in 29, the model then features only a first order transition.

Quantum state diffusion approach We analyze the model (1) by unraveling the master equation with quantum state diffusion [29], that is we express the density operator $\rho$ as the average over stochastic pure states [20, 21]. In contrast to the cooperative resonance fluorescence model, due to the additional $J_{z}$ dissipator, the resulting stochastic Schrödinger equation preserves spin coherent states for any $j$, that is, for any system size 29, 30. The spin coherent states are defined as

$$
\left.|\mu\rangle=\frac{\| \mu\rangle}{\sqrt{\langle\mu|| \mu\rangle}}, \quad \| \mu\right\rangle=\exp \left(\mu J_{-}\right)|j\rangle,
$$

and the density operator is obtained by averaging over the stochastically evolving spin coherent states $\rho(t)=$ $\mathbb{E}(|\mu(t)\rangle\langle\mu(t)|)$ where the complex labels $\mu(t)$ are stochastic trajectories obeying the classical Langevin equation

$$
\mathrm{d} \mu=\left(-\mathrm{i} \frac{\omega}{2}\left(1-\mu^{2}\right)-\tilde{\kappa} \mu\right) \mathrm{d} t+\sqrt{\frac{\kappa}{j}} \mu^{2} \mathrm{~d} \xi_{+}-\sqrt{\frac{\kappa}{j}} \mu \mathrm{d} \xi_{z},
$$

with complex Ito increments $\mathbb{E}\left(\mathrm{d} \xi_{\alpha}\right)=0, \mathrm{~d} \xi_{\alpha} \mathrm{d} \xi_{\beta}^{*}=$ $\delta_{\alpha \beta} \mathrm{d} t$. A rescaled coupling is introduced as $\tilde{\kappa}=\kappa\left(1-\frac{1}{2 j}\right)$. That spin coherent states are preserved is remarkable [30, and reflects the localization property of quantum state diffusion [22. It allows to solve this model particularly easy. Nevertheless, in the following we see that this model features qualitatively the same physics as the cooperative resonance fluorescence model. Unlike meanfield or semi-classical approaches, Eq. (3) provides the exact solution of the master equation (1) for any system size.

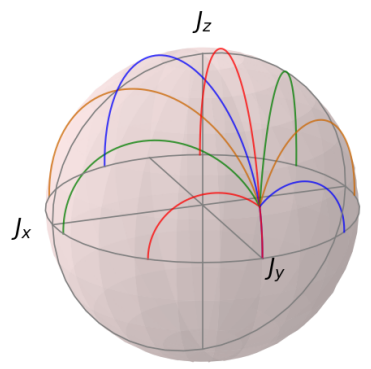

(a) $\lambda=0.95$

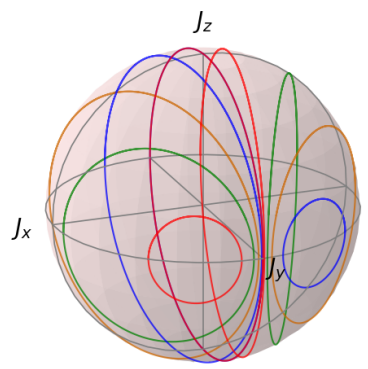

(b) $\lambda=1.05$
FIG. 2: Deterministic trajectories Eq. (4) displayed on the sphere with initial conditions on the equator. In case (a) $(\lambda<1)$ all trajectories flow to a stable fixed point. In case (b) $(\lambda>1)$ solutions are cyclic.

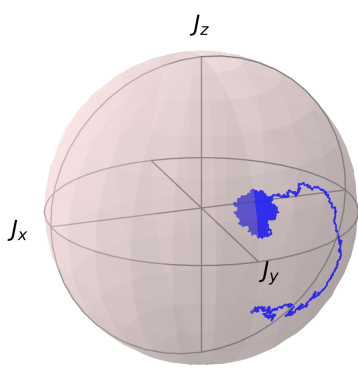

(a) $\lambda=0.95$

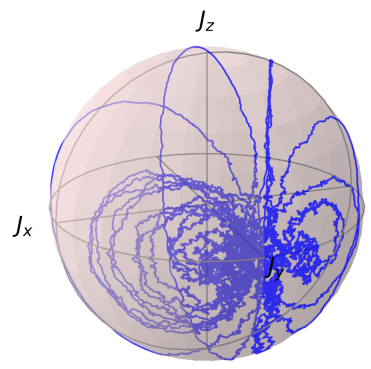

(b) $\lambda=1.05$
FIG. 3: Example of a noisy trajectory Eq. (3) for $j=500$. At $\lambda<1$ (a) a trajectory starting at the unstable fixed point evolves to the stable fixed point and remains there. At $\lambda>1$ (b) a trajectory starting at the right fixed point hops stocastically between the deterministic tori depicted in Fig. 2 (b), such that it eventually explores the entire phase space.

Solution in the thermodynamic limit Neglecting the noise terms in Eq. (3) results in the deterministic equation

$$
\dot{\mu}=-\mathrm{i} \frac{\omega}{2}\left(1-\mu^{2}\right)-\tilde{\kappa} \mu .
$$

Since the strength of fluctuations is $\kappa / j$ this is valid for times much smaller than $j / \kappa$. In particular, it captures the thermodynamic limit $j \rightarrow \infty$. The resulting approximate solutions of Eq. (1) are coherent states with deterministic label $\mu$ obeying Eq. (4). These pure states are just the so called robust states of the master equation [31. At this point we already note that for any finite $j$ the asymptotic state $\rho(t=\infty)$ of Eq. (1) may nevertheless be highly mixed, as will be further elaborated later. The solution of Eq. (4) can be found analytically [29]

$$
\mu_{\mathcal{M}}(t)=\frac{\mu_{-} \sqrt{1+\mathcal{M}} \mathrm{e}^{\mathrm{i} \phi(t)}-\mu_{+} \sqrt{1-\mathcal{M}}}{\sqrt{1+\mathcal{M}} \mathrm{e}^{\mathrm{i} \phi(t)}-\sqrt{1-\mathcal{M}}},
$$


with the phase $\phi(t)=\sqrt{\lambda^{2}-1} \tilde{\kappa} t+\phi(0) . \mathcal{M}(|\mathcal{M}| \leq 1)$ and $\phi(0)$ are determined by the initial condition. There are two fixed points

$$
\mu_{ \pm}=-i \frac{\tilde{\kappa}}{\omega}\left(1 \pm \sqrt{1-\lambda^{2}}\right)
$$

corresponding to $\mathcal{M}= \pm 1$. The most elegant way of displaying the trajectories is to map the complex label $\mu$ to a point on the sphere via the inverse stereographic projection given by

$$
\vec{n}=\langle\mu|\vec{J}| \mu\rangle / j
$$

Some of the deterministic trajectories are depicted in Fig. 2 for two values of $\lambda$. For $\lambda<1$ all trajectories flow to the stable fixed point $\mu_{-}$. In contrast, the solutions are periodic for $\lambda>1$, i.e. $\mu_{\mathcal{M}}(t)$ traverses a closed torus with period $T=2 \pi\left(\tilde{\kappa} \sqrt{\lambda^{2}-1}\right)^{-1}$. As a consequence of the existence of periodic solutions, the spectrum of the Lindbladian becomes gapless and the imaginary parts of the eigenvalues are separated by the fundamental frequency $2 \pi / T$, as observed in 26. Clearly, the existence of two distinct phases in the thermodynamic limit is evident. In the case $\lambda<1$ there is a unique stable (unstable) steady state which is the coherent state $\rho_{-}=\left|\mu_{-}\right\rangle\left\langle\mu_{-}\right|$ $\left(\rho_{+}=\left|\mu_{+}\right\rangle\left\langle\mu_{+}\right|\right)$. For $\lambda>1$ a steady state can be associated with each torus labeled with $\mathcal{M}$, by time averaging the cyclic evolution over one period

$$
\rho_{\mathcal{M}}=\frac{1}{T} \int_{0}^{T} \mathrm{~d} t\left|\mu_{\mathcal{M}}(t)\right\rangle\left\langle\mu_{\mathcal{M}}(t)\right| .
$$

The emergence of periodic solutions has led to the idea that such a phase transition can be associated with a spontaneous breaking of continuous time translation symmetry 26]. Here, however, we can clearly see that the mirror symmetry of the Lindblad generator (1) is spontaneously broken in the $\lambda>1$ phase. The steady states $\rho_{\mathcal{M}}$ in Eq. (8) are not mirror symmetric, since the mirrored state is $\rho_{-\mathcal{M}}[32$. Thus the phase transition happens in presence of an ordinary symmetry breaking [10]. Note that all steady states $\rho_{\mathcal{M}}$ have a vanishing $J_{z}$ expectation value and thus the steady state value of $J_{z}$ is well defined

$$
\frac{\left\langle J_{z}\right\rangle}{j}= \begin{cases}\sqrt{1-\lambda^{2}} & , \lambda<1 \\ 0 & , \lambda>1\end{cases}
$$

indicating a second order phase transition. When in the Hamiltonian an additional symmetry breaking term $\omega_{z} J_{z}$ is present there are no cyclic solutions and a stable fixed point exists for all $\lambda$. The system is then no longer critical at $\lambda=1$ [29].

Finite system size If the system has a finite size $j<\infty$ there always exists a unique steady state which is the asymptotic solution of (1). The uniqueness is seemingly at odds with the previous investigation in the ther- modynamic limit, where for $\lambda>1$ we found a whole family of steady states. The explanation goes as follows. Neglecting the noise terms, as we did in Eq. (4), is only valid for times much shorter than $j / \kappa$. On longer timescales the noise will lead to a mixing process which results in a unique steady state. In Fig. 3 two quantum trajectories are displayed in the two regimes. For $\lambda<1$ there exists a stable fixed point and a weak $(1 / j)$ noise will only cause small fluctuations around this point, see Fig. 3 (a). On the other hand, in the presence of cyclic solutions, when $\lambda>1$, the noise introduces a hopping between neighboring tori, see Fig. 3 (b). Torus hopping allows a single trajectory to eventually explore the entire phase space. In Fig. 4 (a) the $J_{z}$ variance of the unique steady state is displayed for different values of $j$. Clearly, the variance increases with $\lambda$ and for large system sizes approaches a curve which is non-analytic at $\lambda=1$. With the trajectories displayed in Fig. 3 this can be understood in an intuitive way. Moreover, we can even compute analytically the asymptotic curve displayed in Fig. 4 (a). To this aim it is useful to switch to the "action-angle" variables $(\mathcal{M}, \phi)$ used for the deterministic trajectories (5). As elaborated in 29], averaging over the fast dynamics of the angle variable [25, 33, 34] results in a one dimensional stochastic evolution for the torus label $\mathcal{M}$, reflecting the slow torus-hopping process. A stationary distribution for this process can be found analytically

$$
P(\mathcal{M})=\frac{1}{2} \frac{\sqrt{1+2 \lambda^{2}}}{\tanh ^{-1}\left(\left(1+2 \lambda^{2}\right)^{-1 / 2}\right)} \frac{1}{2 \lambda^{2}+1-\mathcal{M}^{2}} .
$$

The unique steady state resulting from the mixing induced by the torus hopping is

$$
\rho_{S S}=\int_{-1}^{1} \mathrm{~d} \mathcal{M} P(\mathcal{M}) \rho_{\mathcal{M}},
$$

with $\rho_{\mathcal{M}}$ from (8). Naturally, this unique state does have the same symmetries as the Lindblad generator, since $P(\mathcal{M})$ is a symmetric distribution. We note in passing that the additional $J_{z}$ dissipator of the present model leads to a different distribution of tori compared to the one derived by Carmichael using a Glauber $P$ distribution technique in 33]. The $J_{z}$ variance of $\rho_{S S}$ can be given in closed form,

$$
\frac{\Delta J_{z}^{2}}{j^{2}}=\left(\lambda^{2}-1\right)\left(\frac{\tanh ^{-1}\left(\sqrt{3}\left(1+2 \lambda^{2}\right)^{-1 / 2}\right)}{\sqrt{3} \tanh ^{-1}\left(\left(1+2 \lambda^{2}\right)^{-1 / 2}\right)}-1\right),
$$

displayed as the dashed line in Fig. 4 (a). Interestingly, this function does not behave like a power law for $\lambda$ close to one. With $\lambda=1+\varepsilon$ we find that the asymptotic curve behaves as $-\varepsilon \ln \varepsilon[29$. This behaviour is hard to find from extrapolating finite size calculations, as can be seen in Fig. 4 (b). There we plot "exponents" $\beta$ that one would associate to the limiting curve by assuming 


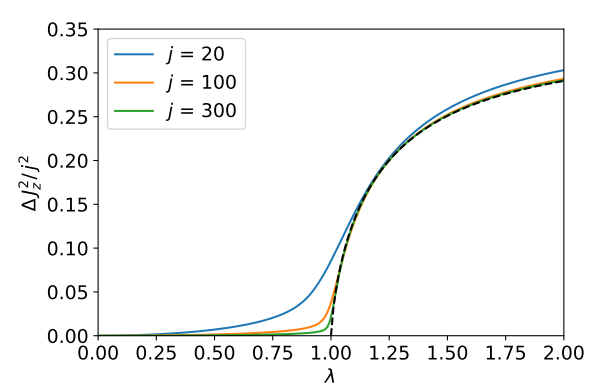

(a) $J_{z}$ variance.

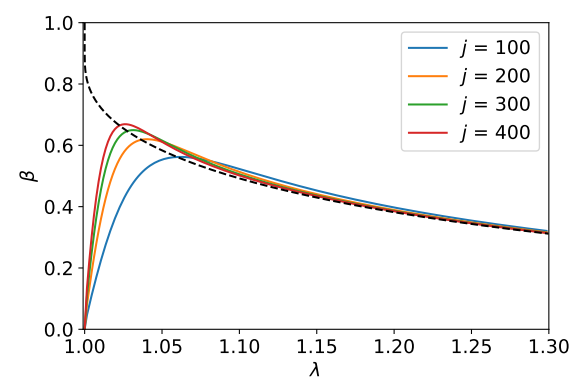

(b) Power law "exponent" $\beta$ of the $J_{z}$ variance.

FIG. 4: Variance of $J_{z}$ in the steady state of master equation (1) as a function of the parameter $\lambda$ for different values of $j(\mathrm{a})$. The dashed line is the asymptotic curve for large $j$ from 12 . The assignment of a critical exponent close to $\lambda=1$ from finite system calculations (b) fails because the asymptotic curve does not behave like a power law in the vicinity of the transition.

a power law, estimated for different values of $\lambda=1+\varepsilon$. Clearly we see from the exact curve that close to $\lambda=1$ no exponent can be associated, and the peculiar behaviour $\beta \simeq 1+\frac{1}{\ln (\varepsilon)}$ with an infinite negative slope at the critical point follows 29].

In the thermodynamic limit different steady states (8) do not have the same $J_{z}$ variance, and thus this quantity is not well defined. As a consequence, this observable does not have a universal power law scaling close to the transition point - see [29] for more details. Fig. 4] (b) manifests also the non-commutativity of the two types of limiting procedures involved. The non universal behaviour is a finite system size effect which emerges when first the stationary state is found and only afterwards the limit of large system size is taken. In order to correctly understand the phase transition, the order of limits has to be interchanged. Neglecting the $1 / j$-terms, as in (4), corresponds to first taking the strict thermodynamic limit leading to the family of steady states (8). Naively computing the steady state of (1) for finite $j$ results always in a unique steady state and the broken symmetry is not revealed.
Critical exponents and finite size scaling Clearly, Eq. (9) gives the critical exponent $1 / 2$ for the $J_{z}$ expectation value. From the exact solution in the thermodynamic limit (5) we can just as easily read off the dynamical critical exponent. Since there is only a single time scale $\xi=\left|1-\lambda^{2}\right|^{-1 / 2} \kappa^{-1}$ the dynamical exponent is also $1 / 2$. With the exact Ito-equations at hand it is also possible to find finite size scaling exponents at $\lambda=1$ by using a renormalization scheme as in [3, 5]. Numerically we observe a finite size power law scaling at the transition point, see Fig. 5. To obtain the exponent analytically we set $\nu=\mu+\mathrm{i}$ and the evolution equation $(3)$ is rewritten as

$$
\mathrm{d} \nu=\mathrm{i} \frac{\tilde{\kappa}}{2} \nu^{2} \mathrm{~d} t+\sqrt{\frac{\kappa}{j}}(\nu-\mathrm{i}) \mathrm{d} \xi_{z}+\sqrt{\frac{\kappa}{j}}(\nu-\mathrm{i})^{2} \mathrm{~d} \xi_{+} .
$$

Under a rescaling of time, close to the transition, $\nu$ has power law scaling.

Indeed, the noiseless equation is invariant under the transformation

$$
t \rightarrow a t, \quad \nu \rightarrow \frac{\nu}{a} .
$$

This transformation changes the evolution equation to 35

$\mathrm{d} \nu=\mathrm{i} \frac{\tilde{\kappa}}{2} \nu^{2} \mathrm{~d} t+a^{3 / 2} \sqrt{\frac{\kappa}{j}}\left(\frac{\nu}{a}-\mathrm{i}\right) \mathrm{d} \xi_{z}+a^{3 / 2} \sqrt{\frac{\kappa}{j}}\left(\frac{\nu}{a}-\mathrm{i}\right)^{2} \mathrm{~d} \xi_{+}$.

For the theory (with fluctuations) to be scale invariant at low frequencies, $j$ must scale as 3 , 5]

$$
\frac{\kappa}{j} \rightarrow \frac{1}{a^{3}} \frac{\kappa}{j}
$$

Then all noise terms containing $\nu / a$ become irrelevant under renormalization and the resulting low frequency theory with constant noise is scale invariant. The finite- $j$ scaling of $\nu$ is found to be $(\kappa / j)^{-\frac{1}{3}} \nu \sim 1$. With $\mu=\nu-\mathrm{i}$, we conclude that for large $j$

$$
\left\langle\mu\left|J_{z}\right| \mu\right\rangle / j \sim \nu+\mathcal{O}\left(\nu^{2}\right) \approx \nu \sim\left(\frac{\kappa}{j}\right)^{\frac{1}{3}} .
$$

As seen in Fig. 5, numerically accessible values match this law quite well.

Conclusions Quantum state diffusion for nonequilibrium quantum dynamics allows us to obtain exact analytical results for a driven dissipative many body quantum system featuring a phase transition. We are able to identify the symmetries of the different phases emerging in the thermodynamical limit, and in this way we can reveal the symmetry breaking associated with the phase transition. For these findings the trajectory picture proves insightful. Moreover, it provides an elegant shortcut to an exact analytical treatment. The physics in the trajectory framework can be characterized 


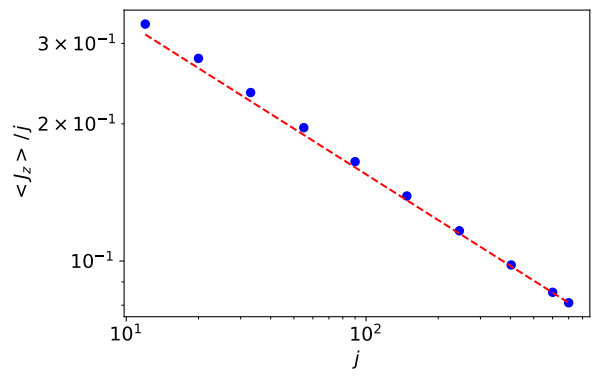

FIG. 5: $J_{z}$ steady state expectation value at $\lambda=1$ for different values of $j$. The dashed line has the predicted slope $-1 / 3$.

by two time scales, $T$ and $j / \kappa$, which describe the deterministic evolution of the open system and the fluctuations, respectively. In the thermodynamic limit $(j \rightarrow \infty)$ the relaxation time scale is infinitely large and the system does not relax, resulting in the degeneracy of the stationary state. Even though we here consider a solvable model in terms of spin coherent states, quantum state diffusion trajectories would reveal the underlying character of the phases also for finite $j$ and for non-linear Hamiltonians. These results strongly support the idea that quantum trajectories help to unravel non-equilibrium phenomena in many body quantum dynamics.

Acknowledgments It is a pleasure to thank Holger Kantz and Konrad Merkel for discussions and advice. V.L. acknowledges support from the International Max Planck Research School (IMPRS) of MPIPKS Dresden.

* valentin.link@tu-dresden.de

† kimmo.luoma@tu-dresden.de

walter.strunz@tu-dresden.de

[1] R. H. Dicke, Phys. Rev. 93, 99 (1954).

[2] F. Dimer, B. Estienne, A. S. Parkins, and H. J. Carmichael, Phys. Rev. A 75, 013804 (2007).

[3] E. G. D. Torre, S. Diehl, M. D. Lukin, S. Sachdev, and P. Strack, Phys. Rev. A 87, 023831 (2013)

[4] R. Gutiérrez-Jáuregui and H. J. Carmichael, Phys. Rev. A 98, 023804 (2018)

[5] M.-J. Hwang, P. Rabl, and M. B. Plenio, Phys. Rev. A 97, 013825 (2018).

[6] K. Baumann, C. Guerlin, F. Brennecke, and T. Esslinger, Nature 464, 1301 (2010).

[7] M. Greiner, O. Mandel, T. Esslinger, T. W. Hänsch, and I. Bloch, Nature 415, 39 (2002)

[8] A. M. Shakirov, Y. E. Shchadilova, A. N. Rubtsov, and P. Ribeiro, Phys. Rev. B 94, 224425 (2016).

[9] J. S. Ferreira and P. Ribeiro, arXiv (2018), 1801.00818

[10] P. Ribeiro and T. Prosen, arXiv (2018), 1807.09438

[11] L. M. Sieberer, M. Buchhold, and S. Diehl, Reports on Progress in Physics 79, 096001 (2016).
[12] L. M. Sieberer, A. Chiocchetta, A. Gambassi, U. C. Täuber, and S. Diehl, Phys. Rev. B 92, 134307 (2015)

[13] J. Raftery, D. Sadri, S. Schmidt, H. E. Türeci, and A. A. Houck, Phys. Rev. X 4, 031043 (2014).

[14] J. Hannukainen and J. Larson, Phys. Rev. A 98, 042113 (2018)

[15] D. Nagy and P. Domokos, Phys. Rev. Lett. 115, 043601 (2015).

[16] E. G. Dalla Torre, E. Demler, T. Giamarchi, and E. Altman, Nat. Phys. 6, 806 (2010)

[17] J. Gelhausen and M. Buchhold, Phys. Rev. A 97, 023807 (2018)

[18] V. Gorini, A. Kossakowski, and E. C. G. Sudarshan, Journal of Mathematical Physics 17, 821 (1976) G. Lindblad, Commun. Math. Phys. 48, 119 (1976).

[19] W. T. Strunz, Journal of Physics A: Mathematical and General 30, 4053 (1997).

[20] N. Gisin and I. C. Percival, J. Phys. A: Math. Gen. 25, $5677(1992)$

[21] M. B. Plenio and P. L. Knight, Rev. Mod. Phys. 70, 101 (1998)

[22] W. T. Strunz and I. C. Percival, Journal of Physics A: Mathematical and General 31, 1801 (1998)

[23] D. F. Walls, P. D. Drummond, S. S. Hassan, and H. J. Carmichael, Progress of Theoretical Physics Supplement 64, 307 (1978).

[24] D. F. Walls, Journal of Physics B: Atomic and Molecular Physics 13, 2001 (1980).

[25] P. Drummond and H. Carmichael, Optics Communications 27, 160 (1978)

[26] F. Iemini, A. Russomanno, J. Keeling, M. Schirò, M. Dalmonte, and R. Fazio, Phys. Rev. Lett. 121, 035301 (2018)

[27] S. Morrison and A. S. Parkins, Journal of Physics B: Atomic, Molecular and Optical Physics 41, 195502 (2008)

[28] S. Schneider and G. J. Milburn, Phys. Rev. A 65, 042107 (2002)

29] See Supplemental Material.

[30] N. Gisin and M. B. Cibils, Journal of Physics A: Mathematical and General 25, 5165 (1992)

[31] W. T. Strunz, in Coherent Evolution in Noisy Environments, edited by A. Buchleitner and K. Hornberger (Springer Berlin Heidelberg, 2008).

[32] The mirror reflected state to a coherent state $|\mu\rangle$ is $\left|-\mu^{*}\right\rangle$.

[33] H. J. Carmichael, Journal of Physics B: Atomic and Molecular Physics 13, 3551 (1980)

[34] A. Kruscha, R. Ketzmerick, and H. Kantz, Phys. Rev. E 85, 066210 (2012)

[35] Ito noise increments scale as $\mathrm{d} \xi \rightarrow \sqrt{a} \mathrm{~d} \xi$. 